\title{
NOISY POLYNOMIAL INTERPOLATION MODULO PRIME POWERS
}

\author{
MAREK KARPINSKI AND IGOR E. SHPARLINSKI
}

\begin{abstract}
We consider the noisy polynomial interpolation problem of recovering an unknown $s$-sparse polynomial $f(X)$ over the ring $\mathbb{Z}_{p^{k}}$ of residues modulo $p^{k}$, where $p$ is a small prime and $k$ is a large integer parameter, from approximate values of the residues of $f(t) \in \mathbb{Z}_{p^{k}}$. Similar results are known for residues modulo a large prime $p$, however the case of prime power modulus $p^{k}$, with small $p$ and large $k$, is new and requires different techniques. We give a deterministic polynomial time algorithm, which for almost given more than a half bits of $f(t)$ for sufficiently many randomly chosen points $t \in \mathbb{Z}_{p^{k}}^{*}$, recovers $f(X)$.
\end{abstract}

\section{INTRODUCTION}

There is a long history and very extensive literature dedicated to algorithms on polynomials in finite fields, see, for example [13]. More recently, there was also increasing interest to algorithms for polynomials over residue rings, especially in residue rings modulo prime powers, see $[5,10,11,14,18,20,33]$ and references therein. Here we continue this directions and consider the noisy polynomial interpolation problem modulo prime powers which is analogue to the same problem in finite fields [30,32], which in turn is an extension of the hidden number problem of Boneh and Venkatesan [2,3].

To be more precise, for an integer $m$ we denore by $\mathbb{Z}_{m}=\mathbb{Z} / m \mathbb{Z}$ the residue ring modulo an integer $m \geqslant 1$, and by $\mathbb{Z}_{m}^{*}$ the group of units of $\mathbb{Z}_{m}$.

Then the noisy polynomial interpolation problem is the problem of finding an unknown $s$-sparse polynomial

$$
f(X)=\sum_{j=1}^{s} a_{j} X^{e_{j}} \in \mathbb{Z}_{m}[X]
$$

Date: November 2, 2020.

2010 Mathematics Subject Classification. 11T71, 11Y16, 68Q25, 68W30.

Key words and phrases. Noisy polynomial interpolation, finite fields, lattice reduction. 
with monomials of degrees $e_{s}>\ldots>e_{1} \geqslant 1$ from approximations to the values of $f(t)$ (treated as integers from the set $\{0,1, \ldots, m-1\}$ ) at polynomially many points $t \in \mathbb{Z}_{m}$ selected uniformly at random.

Several problems of this type are related to the so-called hidden number problem introduced by Boneh and Venkatesan [2,3], which corresponds to a linear polynomial $f(X)=a X$ with unknown $a$, and have already been studied intensively due to their cryptographic relevance, see the survey [31]. For sparse polynomials this problem has been studied in $[30,32]$, for some recent modifications motivated by cryptographic applications, see [12].

More precisely for integers $u$ and $m \geqslant 1$ we denote by $\lfloor s\rfloor_{m}$ the remainder of $s$ on division by $m$.

Furthermore. for integers $s, m \geqslant 1$ and a real $\ell \geqslant 0$ we denote by $\operatorname{MSB}_{\ell, m}(s)$ any integer $u$ such that

$$
\left|\lfloor s\rfloor_{m}-u\right| \leqslant m / 2^{\ell+1} \text {. }
$$

Roughly speaking, $\operatorname{MSB}_{\ell, m}(z)$ gives $\ell$ most significant bits of the remainder on division of $z$ by $m$. However, this definition is more flexible and suits better our purposes. In particular we remark that $\ell$ in the inequality (1.2) is not necessarily an integer.

The sparse polynomial noisy interpolation problem is the problem of finding a polynomial $f(X) \in \mathbb{Z}_{m}[X]$ of the form (1.1) with known exponents $e_{1}, \ldots, e_{s}$ and unknown coefficients $a_{1}, \ldots, a_{s} \in \mathbb{Z}_{m}$. from approximate values of $\lfloor f(t)\rfloor_{m}$ at polynomially many points $t \in \mathbb{Z}_{m}$ selected uniformly at random. We remark that we always assume that the exponents $e_{s}, \ldots, e_{1}$ are positive since if $\ell$ is not very small, it is impossible to distinguish between $f(X)$ and $f(X)+1$.

Here we are interested in the setting where the modulus $m=p^{k}$ is a large power of a fixed prime, for example $m=2^{k}$, while previous works $[2,3,12,30-32]$, address the case when $m=p$ is a large prime. In the case of $m=p^{k}$, we use the ideas of [30,32] combined with new number theoretic tools, coming from [29], and give a polynomial time algorithm provided that for each $t$ slightly more than a half of the bits of $\mathrm{MSB}_{\ell, p^{k}}(f(t))$ are given.

We note that algorithm itself is deterministic, and the only random$n e s s$ is in the choice of the evaluation points $t$, while the consecutive computation is deterministic.

\section{Our Results}

We recall that the notations $U=O(V), U \ll V$ and $V \gg U$ are all equivalent to the assertion that the inequality $|U| \leqslant c|V|$ holds for 
some constant $c>0$, which throughout the paper may depend on the real positive parameters $\eta$ and $\varepsilon$, the integer $s \geqslant 1$ and the prime $p$.

It is also convenient to define $\log z$ as the binary $\operatorname{logarithm}$ of real $z>0$.

We always assume that

$$
n=\lceil k \log p\rceil
$$

is the bit length of the modulus $q=p^{k}$.

Our result depends on the $p$-divisibility of the following determinant, formed by binomial coefficients

$$
\Delta\left(e_{1}, \ldots, e_{s}\right)=\operatorname{det}\left(\begin{array}{ccc}
\left(\begin{array}{c}
e_{1} \\
1
\end{array}\right), & \ldots, & \left(\begin{array}{c}
e_{s} \\
1
\end{array}\right) \\
\ldots, & \ldots, & \ldots \\
\left(\begin{array}{c}
e_{1} \\
s
\end{array}\right), & \ldots, & \left(\begin{array}{c}
e_{s} \\
s
\end{array}\right)
\end{array}\right)=\prod_{i=1}^{s} \frac{e_{i}}{i !} \prod_{1 \leqslant i<j \leqslant s}\left(e_{j}-e_{i}\right) .
$$

Finally, for an integer $a \neq 0$ we denote by $\operatorname{ord}_{p} a$ the $p$-adic order of $a$, that is, the largest integer $\alpha$ with $p^{\alpha} \mid a$ and by

$$
\|a\|_{p}=p^{-\operatorname{ord}_{p} a}
$$

the $p$-adic valuation of $a$.

Theorem 2.1. Let $q=p^{k}$ be a sufficiently large $n$-bit power of a fixed prime $p$ and let $s \geqslant 1$ be a fixed integer. Assume that for the integers $1 \leqslant e_{1}<\ldots<e_{s}<q$ and real $\ell$ we have

$$
\ell \geqslant(0.5+\varepsilon) n \quad \text { and } \quad\left\|\Delta\left(e_{1}, \ldots, e_{s}\right)\right\|_{p} \geqslant s 2^{-\ell(1 /(s+1)-\varepsilon)}
$$

for some fixed $\varepsilon>0$. Then there exists a deterministic polynomial time algorithm $\mathcal{A}$ such that for any polynomial $f(X) \in \mathbb{Z}_{q}[X]$ of the form (1.1), given $2 d$ integers

$$
t_{i} \quad \text { and } \quad w_{i}=\operatorname{MSB}_{\ell, q}\left(f\left(t_{i}\right)\right), \quad i=1, \ldots, d,
$$

where

$$
d=\left\lceil 4(s+1) \varepsilon^{-1}\right\rceil
$$

its output satisfies

$$
\operatorname{Pr}_{t_{1}, \ldots, t_{d} \in \mathbb{Z}_{q}^{*}}\left[\mathcal{A}\left(t_{1}, \ldots, t_{d} ; w_{1}, \ldots, w_{d}\right)=\left(a_{1}, \ldots, a_{s}\right)\right] \geqslant 1-1 / q
$$

if $t_{1}, \ldots, t_{d}$ are chosen uniformly and independently at random from $\mathbb{Z}_{q}^{*}$.

Analysing the proof of Theorem 2.1 one can easily see that the value of $d$ is not optimised and can be improved at the cost of more tedious calculations.

We note that it is natural to expect that any result of the type of Theorem 2.1 should depend on the $p$-divisibility of the determinant 
$\Delta\left(e_{1}, \ldots, e_{s}\right)$, which in turn measures the $p$-adic closeness of the exponents in different monomials of $f$ and thus controls $p$-adic independence of these monomials.

\section{Congruences With SPARSE POLynOmials}

For a polynomial $F \in \mathbb{Z}_{m}[X]$ in a residue ring modulo $m \geqslant 2$, and integers $a$ and $h$, we denote by $N_{F}(a, h ; m)$ the number of solutions to the congruence

$$
F(x) \equiv u \quad(\bmod m), \quad x \in \mathbb{Z}_{m}^{*}, u \in\{a+1, \ldots, a+h\} .
$$

A natural and powerful tool to estimate $N_{F}(a, h ; m)$ is given by bounds on exponential sums

$$
S(F, m)=\sum_{\substack{x=1 \\ \operatorname{gcd}(x, m)=1}}^{m} \mathbf{e}_{m}(F(x))
$$

where

$$
\mathbf{e}_{m}(z)=\exp (2 \pi i z / m) \text {. }
$$

In fact a bound on such sums for a sparse polynomial $F$ as in (1.1) has been given in [29, Theorem 1], which however requires that the $\operatorname{deg} f$ is bounded (independently of $q$ ) and thus makes it is very restrictive for our applications. Bourgain [4] has given different versions of this result and relaxed the condition of $\operatorname{deg} f$ however the corresponding bounds are weaker.

Here we exploit the fact that the results and method of [29] allow us to obtain a bound on $N_{F}(a, h ; q)$ which depends on the $p$-divisibility of $\Delta\left(e_{1}, \ldots, e_{s}\right)$ (which controls $p$-adic properties of the differences between exponents $\left.e_{1}, \ldots, e_{n}\right)$ rather than on $\operatorname{deg} f=\max \left\{e_{1}, \ldots, e_{n}\right\}$.

First we need a slightly modified and explicit version of a result from [29].

Lemma 3.1. Let $q=p^{\alpha}$ be a power of a fixed prime $p$ and let

$$
F(X)=\sum_{j=1}^{s} A_{j} X^{e_{j}} \in \mathbb{Z}_{q}[X]
$$

be a polynomial such that

$$
\operatorname{gcd}\left(A_{1}, \ldots, A_{s}, p\right)=1 \quad \text { and } \quad e_{s}>\ldots>e_{1} \geqslant 1 .
$$

If for some fixed $\eta>0$ we have

$$
p^{\rho} \leqslant s^{-1} q^{\eta}
$$

where

$$
\rho=\operatorname{ord}_{p} \Delta\left(e_{1}, \ldots, e_{s}\right),
$$


then

$$
|S(F, q)| \ll q^{1-1 / s+\eta(s+1) / s} .
$$

Proof. We essentially follow the proof of [29, Lemma 5] and trace the dependence on $\rho$. In particular, as in [29] we fix some $\eta>0$ and define $\beta=\lfloor\alpha \eta\rfloor+1$ and define the integer $m$ by the inequalities

$$
\beta m<\alpha<\beta(m+1) \text {. }
$$

Let us also define the following differential operators

Finally, let

$$
D_{\nu}=\frac{1}{\nu !} \cdot \frac{d^{\nu}}{d x^{\nu}} \quad \nu=0,1, \ldots
$$

$$
\vartheta_{x}=\max \left\{\beta(\nu-1)+\operatorname{ord}_{p} D_{\nu} F(x): \nu=1, \ldots, s\right\} .
$$

By [29, Lemma 2], the inequality

$$
\vartheta_{x} \leqslant \rho+\beta(s-1)
$$

holds, provided that $\operatorname{gcd}(x, p)=1$.

We now note by our assumption on the size of $p^{\rho}$ we have

$$
\beta s-\vartheta_{x} \geqslant \beta-\rho \geqslant \alpha \eta-\rho>\frac{\log s}{\log p},
$$

which ensures that the condition of [29, Lemma 4] is verified, see also [29, Equation (5)]

Then by [29, Equation (6)] we have

$$
|S(F, q)| \leqslant \sum_{\substack{x=1 \\ \operatorname{gcd}(x, p)=1}}^{p^{\beta}}|\sigma(x)|
$$

where

$$
\sigma(x)=\sum_{y=1}^{p^{\alpha-\beta}} \mathbf{e}_{p^{\alpha-\beta}}\left(\sum_{\nu=1}^{m} p^{\beta(\nu-1)} y^{\nu} D_{\nu} F(x)\right) .
$$

We can certainly assume that $\alpha$ is large enough (in terms of $\eta$ ) and thus we are in the case $\alpha-\beta>1$ of the proof of [29, Lemma 5]. In this case, by [29, Equation (8)], we have

$$
\sigma(x)=O\left(p^{\alpha(1-1 / s)}\right)
$$

provided that $\rho$ is fixed. We now trace the dependence on $\rho$, which is explicit in the proof of [29, Lemma 5] till the very last step.

More precisely, it is shown in the proof of [29, Lemma 5] that for $\vartheta_{x} \geqslant \alpha-\beta$ we have

$$
|\sigma(x)| \leqslant p^{\rho+\beta(s-1)}
$$


Hence we see from (3.3) that the total contribution to the bound on $|S(F, q)|$ from all such values of $x$, which we denote by $\Sigma_{1}$, is at most

$$
\Sigma_{1} \leqslant p^{\beta} p^{\rho+\beta(s-1)}=p^{\rho+\beta s} \leqslant p^{\rho+\eta \alpha s+s} .
$$

Furthermore, in the case when $\vartheta_{x}<\alpha-\beta$ it is shown in the proof of [29, Lemma 5] that for some constant $c$ which depends only on $t$ and $m$ (and thus only on $t$ and $\eta$ )

$$
|\sigma(x)| \leqslant c p^{\vartheta_{x}+\left(\alpha-\beta-\vartheta_{x}\right)(1-1 / s)}
$$

and also that the exponent satisfies the inequality

$$
\begin{aligned}
\vartheta_{x}+\left(\alpha-\beta-\vartheta_{x}\right)(1-1 / s) & =\left(\vartheta_{x}-\beta(s-1)\right) / s+\alpha(1-1 / s) \\
& \leqslant \rho / s+\alpha(1-1 / s),
\end{aligned}
$$

which follows from (3.2). Hence we see from (3.3) that the total contribution to the bound on $|S(F, q)|$ from all such values of $x$, which we denote by $\Sigma_{2}$, is at most

$$
\Sigma_{2} \leqslant c p^{\beta} p^{\rho / s+\alpha(1-1 / s)} \leqslant c p^{\rho / s+\alpha(1-1 / s)+\eta \alpha} .
$$

Combining (3.4) and (3.5), we see from (3.3) and the assumed rrestriction of $p^{\rho}$ that

$$
\begin{aligned}
|S(F, q)| & \leqslant \Sigma_{1}+\Sigma_{2} \leqslant p^{\rho+\eta \alpha s+s}+c p^{\rho / s+\alpha(1-1 / s)+\eta \alpha} \\
& \ll p^{\eta \alpha(s+1)}+p^{\eta \alpha / s+\alpha(1-1 / s)+\eta \alpha} \ll q^{\eta(s+1)}+q^{1-1 / s+\eta(s+1) / s} .
\end{aligned}
$$

We can assume that $\eta \leqslant 1 /(s+1)$ as otherwise the second term in the above inequality exceeds the trivial bound $q$. On the other hand for $\eta<1 /(s+1)$ we have

$$
\eta(s+1)<1-1 / s+\eta(s+1) / s .
$$

Hence the second term always dominates and the desired bound follows.

We remark that the bound of exponential sums of [29, Lemma 5], which underlies the proof of Lemma 3.1, is based, in turn or a result of Mit'kin [23, Lemma 1.2]. Using a bound of Cochrane and Zheng [7, Equation (2.11)] one can get better values of implied constants; this however does not affect our main result.

Combining Lemma 3.1 with the classical Erdös-Turán inequality (see, for example, [9, Theorem 1.21]), which links the irregularity of distribution of sequences to exponential sums, we immediately derive that $N_{F}(a, h ; q)$ is close to its expected value

$$
h \frac{\varphi(q)}{q}=h \frac{p-1}{p},
$$


where $\varphi(q)$ is the Euler function. More precisely, we recall that the discrepancy $D(N)$ of a sequence in $\xi_{1}, \ldots, \xi_{N} \in[0,1)$ is defined as

$$
D(N)=\sup _{0 \leqslant \alpha<\beta \leqslant 1}\left|\#\left\{1 \leqslant n \leqslant N: \xi_{n} \in[\alpha, \beta)\right\}-(\beta-\alpha) N\right|,
$$

where $\# \mathcal{S}$ denotes the cardinality of $\mathcal{S}$ (if it is finite), see [9] for background.

By the classical Erdős-Turán inequality (see, for instance, [9, Theorem 1.21]) we have the following estimate of the discrepancy via exponential sums.

Lemma 3.2. Let $\xi_{n}, n \in \mathbb{N}$, be a sequence in $[0,1)$. Then for any $H \in \mathbb{N}$, we have

$$
D(N) \leqslant 3\left(\frac{N}{H+1}+\sum_{h=1}^{H} \frac{1}{h}\left|\sum_{n=1}^{N} \exp \left(2 \pi i h \xi_{n}\right)\right|\right) .
$$

We now interpret the congruence (3.1) as a condition on the fractional parts $\{F(x) / q\}$ to fall in a certain interval of a unit torus $\mathbb{R} / \mathbb{Z} \cong$ $[0,1)$ of length $h / q$. This immediately implies the desired result.

Lemma 3.3. Let $q=p^{\alpha}$ be a power of a fixed prime $p$ and let

$$
F(X)=\sum_{j=1}^{s} A_{j} X^{e_{j}} \in \mathbb{Z}_{q}[X]
$$

be a polynomial such that

$$
\operatorname{gcd}\left(A_{1}, \ldots, A_{s}, p\right)=1 \quad \text { and } \quad e_{s}>\ldots>e_{1} \geqslant 1 .
$$

If for some fixed $\varepsilon>0$ we have

$$
p^{\rho} \leqslant s^{-1} q^{1 /(s+1)-\varepsilon},
$$

where

$$
\rho=\operatorname{ord}_{p} \Delta\left(e_{1}, \ldots, e_{s}\right)
$$

then

$$
\left|N_{F}(a, h ; q)-h \frac{p-1}{p}\right| \ll q^{1-\varepsilon} \log q .
$$

Proof. We set

$$
\eta=\frac{1-(s+1) \varepsilon}{(s+1)(1-\varepsilon)} \quad \text { and } \quad H=\left\lfloor q^{\varepsilon}\right\rfloor .
$$


For each exponential sum which appears in the bound of Lemma 3.2 corresponding to $h$ with $\operatorname{gcd}(h, q)=d$, we have

$$
\sum_{\substack{x=1 \\ \operatorname{gcd}(x, p)=1}}^{q} \mathbf{e}_{q}(h F(x))=d \sum_{\substack{x=1 \\ \operatorname{gcd}(x, p)=1}}^{q} \mathbf{e}_{q / d}((h / d) F(x))
$$

Furthermore, for each $h=1, \ldots, H$ we note that

$$
s^{-1}(q / d)^{\eta} \geqslant s^{-1}(q / H)^{\eta} \geqslant s^{-1}\left(q^{1-\varepsilon}\right)^{\eta}=s^{-1} q^{1 /(s+1)-\varepsilon} \geqslant p^{\rho} .
$$

We also observe that

$$
1 / s-\eta(s+1) / s=\frac{1}{s}(1-\eta(s+1))=\frac{1}{s}\left(1-\frac{1-(s+1) \varepsilon}{1-\varepsilon}\right)=\frac{\varepsilon}{1-\varepsilon} .
$$

We see from (3.7) that we can now apply Lemma 3.1 to the sum on the right hand side of (3.6) with the above $\eta$. Thus we taking into account our calculation in (3.8), we obtain

$$
\begin{aligned}
\sum_{\substack{x=1 \\
\operatorname{gcd}(x, p)=1}}^{q} \mathbf{e}_{q}(h F(x)) & \ll d(q / d)^{1-1 / s+\eta(s+1) / s}=q(q / d)^{-1 / s+\eta(s+1) / s} \\
& =q(q / d)^{-\varepsilon /(1-\varepsilon)} \leqslant q\left(q^{1-\varepsilon}\right)^{-\varepsilon /(1-\varepsilon)}=q^{1-\varepsilon}
\end{aligned}
$$

Therefore, Lemma 3.2 now yields

$$
\left|N_{F}(a, h ; q)-h \frac{p-1}{p}\right| \ll q^{1-\varepsilon} \log q .
$$

which concludes the proof.

Finally, it is convenient to have an upper bound on $N_{F}(a, h ; q)$ for polynomials with non-necessary co-prime with $p$ coefficients. Namely if for $F$ as in Lemma 3.3 we have

$$
\operatorname{gcd}\left(A_{1}, \ldots, A_{s}, q\right)=D
$$

then provided $h \leqslant q / D$ we have

$$
N_{F}(a, h ; q)=D N_{D^{-1} F}(a, h ; q / D) .
$$

Corollary 3.4. Let $q=p^{\alpha}$ be a power of a fixed prime $p$ and let

$$
F(X)=\sum_{j=1}^{s} A_{j} X^{e_{j}} \in \mathbb{Z}_{q}[X]
$$

be a polynomial such that

$$
\operatorname{gcd}\left(A_{1}, \ldots, A_{s}, q\right)=D \quad \text { and } \quad e_{s}>\ldots>e_{1} \geqslant 1 .
$$


If for some fixed $\varepsilon>0$ we have

$$
p^{\rho} \leqslant s^{-1}(q / D)^{1 /(s+1)-\varepsilon},
$$

where

$$
\rho=\operatorname{ord}_{p} \Delta\left(e_{1}, \ldots, e_{s}\right)
$$

then

$$
N_{F}(a, h ; q) \leqslant D h+D^{\varepsilon} q^{1-\varepsilon} \log q .
$$

Note that in Corollary 3.4 we have abandoned the condition $h \leqslant q / D$ which is needed for (3.9) as for $h>q / D$ its bound is trivial.

\section{BACKGROUND ON LATTICES}

As in $[2,3]$, and then in $[30,32]$, our results rely on some lattice algorithms. We therefore review some relevant results and definitions, we refer to $[8,16,17]$ for more details and the general theory.

Let $\left\{\mathbf{b}_{1}, \ldots, \mathbf{b}_{N}\right\}$ be a set of $N$ linearly independent vectors in $\mathbb{R}^{N}$. The set of vectors

$$
\mathcal{L}=\left\{\mathbf{z}: \mathbf{z}=\sum_{i=1}^{N} c_{i} \mathbf{b}_{i}, \quad c_{1}, \ldots, c_{N} \in \mathbb{Z}\right\}
$$

is called an $s$-dimensional full rank lattice.

The set $\left\{\mathbf{b}_{1}, \ldots, \mathbf{b}_{N}\right\}$ is called a basis of $\mathcal{L}$.

The volume of the parallelepiped defined by the vectors $\mathbf{b}_{1}, \ldots, \mathbf{b}_{N}$ is called the volume of the lattice and denoted by $\operatorname{Vol}(\mathcal{L})$. Typically, lattice problems are easier when the Euclidean norms of all basis vectors are close to $\operatorname{Vol}(\mathcal{L})^{1 / N}$.

Let $\|\mathbf{z}\|$ denote the standard Euclidean norm in $\mathbb{R}^{N}$.

One of the most fundamental problems in this area is the closest vector problem, CVP: given a basis of a lattice $\mathcal{L}$ in $\mathbb{R}^{N}$ and a target vector $\mathbf{u} \in \mathbb{R}^{N}$, find a lattice vector $\mathbf{v} \in \mathcal{L}$ which minimizes the Euclidean norm $\|\mathbf{u}-\mathbf{v}\|$ among all lattice vectors. It is well know that CVP is NP-hard when the dimension $N \rightarrow \infty$ (see [22,24-27] for references).

There are several approximate algorithms to find vectors in lattices which are close to a given target vector $\mathbf{r}=\left(r_{1}, \ldots, r_{N}\right) \in \mathbb{R}^{N}$, see $[1$, $19,28]$ which build on the classical lattice basis reduction algorithm of Lenstra, Lenstra and Lovász [21], we also refer to [24-27] for possible improvements and further references.

However, it is important to observe that in our case, the dimension of the lattice is bounded so we can use one of the deterministic algorithms which finds the closest vector exactly. For example, we appeal to the following result of Micciancio and Voulgaris [22, Corollary 5.6]. 
Lemma 4.1. Assume that we are given a basis of a lattice $\mathcal{L}$, which consists of vectors of rational numbers $\mathbf{b}_{1}, \ldots, \mathbf{b}_{N} \in \mathbb{Q}^{N}$ and a vector $\mathbf{r} \in \mathbb{Q}^{N}$ such that their numerators and denominators of $\mathbf{b}_{1}, \ldots, \mathbf{b}_{N}, \mathbf{r}$ are at most $n$-bits long. There is a deterministic algorithm which for a fixed $N$, in time polynomial in $n$, finds a lattice vector $\mathbf{v}=$ $\left(v_{1}, \ldots, v_{N}\right) \in \mathcal{L}$ satisfying the inequality

$$
\|\mathbf{v}-\mathbf{r}\|=\min \{\|\mathbf{v}-\mathbf{z}\|: \mathbf{z} \in \mathcal{L}\}
$$

\section{LATTiCES AND POLYNOMiAL APPROXIMATIONS}

Let $\mathbf{e}=\left(e_{1}, \ldots, e_{s}\right)$.

For $t_{1}, \ldots, t_{d} \in \mathbb{Z}_{q}$, we denote by $\mathcal{L}_{\mathbf{e}, q}\left(t_{1}, \ldots, t_{d}\right)$ the $(d+s)$-dimensional lattice generated by the rows of the following $(d+s) \times(d+s)$-matrix

$$
\left(\begin{array}{ccccccc}
q & 0 & \ldots & 0 & 0 & \ldots & 0 \\
0 & q & \ldots & 0 & 0 & \ldots & 0 \\
\vdots & & \ddots & & \vdots & & \vdots \\
0 & 0 & \ldots & q & 0 & \ldots & 0 \\
t_{1}^{e_{1}} & t_{2}^{e_{1}} & \ldots & t_{d}^{e_{1}} & 1 / 2^{n+1} & \ldots & 0 \\
\vdots & & & & \vdots & \ddots & \vdots \\
t_{1}^{e_{s}} & t_{2}^{e_{s}} & \ldots & t_{d}^{e_{s}} & 0 & \ldots & 1 / 2^{n+1}
\end{array}\right)
$$

The following result is a generalization of several previous results of similar flavour obtained for a large prime number $q=p$, see $[2,30,32]$.

Lemma 5.1. Let $q=p^{k}$ be a sufficiently large $n$-bit power of $p$ and let $s \geqslant 1$ be a fixed integer. Let $f(X) \in \mathbb{Z}_{q}[X]$ be a polynomial of the form (1.1) with known exponents $1 \leqslant e_{1}<\ldots<e_{s}<q$. If the conditions (2.1) hold then for

$$
d=\left\lceil 2(s+1) \varepsilon^{-1}\right\rceil,
$$

t the following holds. If $t_{1}, \ldots, t_{d} \in \mathbb{Z}_{q}^{*}$ are chosen uniformly and independently at random, then with probability $P \geqslant 1-1 / q$ for any vector $\mathbf{u}=\left(u_{1}, \ldots, u_{d}, 0, \ldots, 0\right)$ with

$$
\left(\sum_{i=1}^{d}\left(\left\lfloor f\left(t_{i}\right)\right\rfloor_{q}-u_{i}\right)^{2}\right)^{1 / 2} \leqslant 2^{-\ell} q
$$

all vectors

$$
\mathbf{v}=\left(v_{1}, \ldots, v_{d}, v_{d+1}, \ldots, v_{d+s}\right) \in \mathcal{L}_{\mathbf{e}, q}\left(t_{1}, \ldots, t_{d}\right)
$$


satisfying

$$
\left(\sum_{i=1}^{d}\left(v_{i}-u_{i}\right)^{2}\right)^{1 / 2} \leqslant 2^{-\ell} q
$$

are of the form

$$
\mathbf{v}=\left(\left\lfloor\sum_{j=1}^{s} b_{j} t_{1}^{e_{j}}\right\rfloor_{q}, \ldots,\left\lfloor\sum_{j=1}^{s} b_{j} t_{d}^{e_{j}}\right\rfloor_{q}, b_{1} / 2^{k+1}, \ldots, b_{s} / 2^{k+1}\right)
$$

with some integers $b_{j} \equiv a_{j}(\bmod q), j=1, \ldots, s$.

Proof. As in [2] we define the modular distance between two integers $\beta$ and $\gamma$ as

$$
\begin{aligned}
\operatorname{dist}_{q}(\beta, \gamma) & =\min _{b \in \mathbb{Z}}|\beta-\gamma-b q| \\
& =\min \left\{\lfloor\beta-\gamma\rfloor_{q}, q-\lfloor\beta-\gamma\rfloor_{q}\right\} .
\end{aligned}
$$

Let $\mathcal{P}_{f}$ denote the set of $q^{s}-1$ polynomials

$$
g(X)=\sum_{j=1}^{s} b_{j} X^{e_{j}} \in \mathbb{Z}_{q}[X]
$$

with $g \neq f$.

For a polynomial $g \in \mathcal{P}_{f}$ we denote by $P(g)$ the probability that

$$
\operatorname{dist}_{q}(f(t), g(t)) \leqslant 2^{-\ell+1} q,
$$

for $t \in \mathbb{Z}_{q}^{*}$ selected uniformly at random. To estimate $P(g)$ we consider the polynomial

$$
F(X)=f(X)-g(X)=\sum_{j=1}^{s} A_{j} X^{e_{j}} \in \mathbb{Z}_{q}[X] .
$$

Clearly, $F(X)$ is not identical to zero in $\mathbb{Z}_{q}$. Hence, if (5.2) is possible for some $t \in \mathbb{Z}_{q}^{*}$, then for

$$
D=\operatorname{gcd}\left(A_{1}, \ldots, A_{s}, q\right)
$$

we have

$$
D \leqslant 2^{-\ell+1} q .
$$

Therefore, by our assumption, we have

$$
s^{-1}(q / D)^{1 /(s+1)-\varepsilon} \geqslant s^{-1} 2^{\ell(1 /(s+1)-\varepsilon)} \geqslant p^{\rho},
$$

and thus Corollary 3.4 applies.

We now set

$$
a=-\left\lfloor 2^{-\ell+1} q\right\rfloor \quad \text { and } \quad h=2\left\lceil 2^{-\ell+1} q\right\rceil+1 .
$$


We see from Corollary 3.4 that

$$
P(g)=\frac{1}{\varphi(q)} N_{F}(a, h ; q) \leqslant \frac{1}{\varphi(q)}\left(D h+D^{\varepsilon} q^{1-\varepsilon} \log q\right) .
$$

Hence, recalling the bound (5.4) and the choice of $h$ in (5.5) we obtain

$$
P(g) \ll 2^{-2 \ell} q+2^{-\ell \varepsilon} \log q .
$$

Recalling the inequalities (2.1), we obtain

$$
2^{-2 \ell} q \ll 2^{n-2 \ell} \ll 2^{-2 \varepsilon n} \ll q^{-2 \varepsilon}
$$

and

$$
2^{-\ell \varepsilon} \log q \ll q^{(1 / 2+\varepsilon) \varepsilon)} \log q \leqslant q^{-\varepsilon / 2} .
$$

Hence

$$
P(g) \ll q^{-\varepsilon / 2}
$$

provided that $q$ is large enough.

Therefore, for any $g \in \mathcal{P}_{f}$,

$$
\begin{array}{r}
\operatorname{Pr}\left[\exists i \in[1, d] \mid \operatorname{dist}_{q}\left(g\left(t_{i}\right), f\left(t_{i}\right)\right)>2^{-\ell+1} p\right] \\
=1-P(g)^{d} \geqslant 1-q^{-d \varepsilon / 2},
\end{array}
$$

where the probability is taken over $t_{1}, \ldots, t_{d} \in \mathbb{Z}_{q}$ chosen uniformly and independently at random.

Since $\# \mathcal{P}_{f}=q^{s}-1$, taking

$$
d=\left\lceil 2(s+1) \varepsilon^{-1}\right\rceil
$$

we obtain

$$
\begin{aligned}
\operatorname{Pr}\left[\forall g \in \mathcal{P}_{f}, \exists i \in[1, d] \mid \operatorname{dist}_{q}\left(g\left(t_{i}\right), f\left(t_{i}\right)\right)\right. & \left.>2^{-\ell+1} q\right] \\
& \geqslant 1-\left(q^{s}-1\right) q^{-d \varepsilon / 2}>1-1 / q,
\end{aligned}
$$

provided that $q$ is large enough.

The rest of the proof is essentially identical to the proof of [2, Theorem 5], see also the proof of [30, Theorem 8]. Indeed, we fix some integers $t_{1}, \ldots, t_{d}$ with

$$
\min _{g \in \mathcal{P}_{f}} \max _{i \in[1, d]} \operatorname{dist}_{q}\left(g\left(t_{i}\right), f\left(t_{i}\right)\right)>2^{-\ell+1} q .
$$

Let $\mathbf{v} \in \mathcal{L}_{\mathbf{e}, q}\left(t_{1}, \ldots, t_{d}\right)$ be a lattice point satisfying

$$
\left(\sum_{i=1}^{d}\left(v_{i}-u_{i}\right)^{2}\right)^{1 / 2} \leqslant 2^{-\ell} q
$$


Clearly, since $\mathbf{v} \in \mathcal{L}_{\mathbf{e}, q}\left(t_{1}, \ldots, t_{d}\right)$, there are some integers $\beta_{1}, \ldots, \beta_{s}$ and $z_{1}, \ldots, z_{d}$ such that

$$
\mathbf{v}=\left(\sum_{j=1}^{s} \beta_{j} t_{1}^{e_{j}}-z_{1} q, \ldots, \sum_{j=1}^{s} \beta_{j} t_{d}^{e_{j}}-z_{d} q, \beta_{1} / 2^{k+1}, \ldots, \beta_{s} / 2^{k+1}\right) .
$$

If $\beta_{j} \equiv \alpha_{j}(\bmod q), j=1, \ldots, s$, then for all $i=1, \ldots, d$ we have

$$
\sum_{j=1}^{s} \beta_{j} t_{i}^{e_{j}}-z_{i} q=\left\lfloor\sum_{j=1}^{s} \beta_{j} t_{i}^{e_{j}}\right\rfloor_{q}=\left\lfloor f\left(t_{i}\right)\right\rfloor_{q},
$$

since otherwise there is $i \in[1, d]$ such that $\left|v_{i}-u_{i}\right|>2^{-\ell} q$.

Now suppose that $\beta_{j} \not \equiv \alpha_{j}(\bmod q)$ for some $j \in[1, s]$. In this case we have

$$
\begin{aligned}
\left(\sum_{i=1}^{d}\left(v_{i}-u_{i}\right)^{2}\right)^{1 / 2} & \geqslant \min _{i \in[1, d]} \operatorname{dist}_{q}\left(\sum_{j=1}^{s} \beta_{j} t_{i}^{e_{j}}, u_{i}\right) \\
& \geqslant \min _{i \in[1, d]} \operatorname{dist}_{q}\left(f\left(t_{i}\right), \sum_{j=1}^{s} \beta_{j} t_{i}^{e_{j}}\right)-\operatorname{dist}_{q}\left(u_{i}, f\left(t_{i}\right)\right) \\
& >2^{-\ell+1} q-2^{-\ell} q=2^{-\ell} q,
\end{aligned}
$$

that contradicts our assumption. As we have seen, the condition (5.6) holds with probability exceeding $1-2^{-\ell}$ and the result follows.

\section{Proof of Theorem 2.1}

As in all previous works, we follow the same arguments as in the proof of of [2, Theorem 1] which we briefly outline here for the sake of completeness. We refer to the first $d$ vectors in the matrix (5.1) as $q$-vectors and we refer to the other $s$ vectors as power-vectors.

We recall (2.2) and consider the vector

$$
\mathbf{w}=\left(w_{1}, \ldots, w_{d}, w_{d+1}, \ldots, w_{d+s}\right)
$$

where

$$
w_{d+j}=0, \quad j=1, \ldots, s .
$$

We can certainly assume that

$$
\ell \leqslant n
$$

as otherwise the result is trivial. Then multiplying the $j$ th powervector of the matrix (5.1) by $\alpha_{j}$ and subtracting a certain multiple of the $j$ th $q$-vector, $j=1, \ldots, s$, we obtain a lattice point

$$
\mathbf{u}_{f}=\left(u_{1}, \ldots, u_{d}, \alpha_{1} / 2^{n+1}, \ldots, \alpha_{s} / 2^{n+1}\right) \in \mathcal{L}_{\mathbf{e}, q}\left(t_{1}, \ldots, t_{d}\right)
$$


such that

$$
\left|u_{i}-w_{i}\right|<q 2^{-\ell-1}, \quad i=1, \ldots, d+s,
$$

where $u_{d+j}=\alpha_{j} / 2^{n+1}, j=1, \ldots, s$. Therefore,

$$
\sum_{i=1}^{d+s}\left(u_{i}-w_{i}\right)^{2} \leqslant(d+s) 2^{-2 \ell-2} q^{2} .
$$

We can assume that $q$ is large enough so that $s \leqslant n$. Therefore $d+s=O(n)$. Now we can use Lemma 4.1 to find in polynomial time a lattice vector $\mathbf{v}=\left(v_{1}, \ldots, v_{d}, v_{d+1}, \ldots, v_{d+s}\right) \in \mathcal{L}_{\mathbf{e}, q}\left(t_{1}, \ldots, t_{d}\right)$ such that

$$
\begin{aligned}
\sum_{i=1}^{d}\left(v_{i}-w_{i}\right)^{2} & =\min \left\{\sum_{i=1}^{d+s}\left(z_{i}-w_{i}\right)^{2}, \quad \mathbf{z}=\left(z_{1}, \ldots, z_{d+s}\right)\right\} \\
& \leqslant(d+s) 2^{-2 \ell-2} q^{2} \leqslant 2^{-2 \ell_{0}-2} q^{2}
\end{aligned}
$$

provided that $q$ is large enough, where, for example, we can choose

$$
\ell_{0}=(0.5+\varepsilon / 2) n \text {. }
$$

Applying Lemma 5.1 with $\ell_{0}$ in place of $\ell$, and thus with $\varepsilon / 2$ in place of $\varepsilon$ we see that $\mathbf{v}=\mathbf{u}_{f}$ with probability at least $1-1 / q$, and therefore the coefficients of $f$ can be recovered in polynomial time.

\section{Comments}

It seems like a natural idea to classify polynomials $g \in \mathcal{P}_{f}$ in the proof of Theorem 2.1 depending on the size of $D=\operatorname{gcd}\left(A_{1}, \ldots, A_{s}, q\right)$ where $A_{1}, \ldots, A_{s}$ are as in (5.3), instead of using the worst case bound (5.4) We can then take into account that for a given $D=p^{r}$ there are at most $\left(q / p^{r}\right)^{s}$ polynomials $g \in \mathcal{P}_{f}$ with this values of $D$. Unfortunately, this approach may only help to reduce slightly the value of $d$ in Theorem 2.1, which is not optimised anyway.

We remark that here we essentially consider the interpolation problem when the values of a polynomial $f$ are corrupted by an additive noise. That is, for any $t \in \mathbb{Z}_{q}^{*}$ we are given $f(t)+\vartheta$ for some $\vartheta \in \mathbb{Z}_{q}$ which is not too large. For a large prime $q=p$, in [15] the case of multiplicative noise has been studied, where for any $t \in \mathbb{Z}_{q}^{*}$ we are given the residue modulo $q$ of $\rho f(t)$ for some rational $\rho$ with not too large numerator and denominator. It is certainly interesting to consider this scenario with multiplicative noise modulo powers of small primes as in this work. 


\section{ACKNOWLEDGEMENT}

This work started during a very enjoyable visit of the second author to the University of Bonn, whose hospitality is very much appreciated. This visit was supported by the excellence grant EXC 2-1 of the Hausdorff Center for Mathematics.

During the preparation of this work the first author was supported in part by the Deutsche Forschungsgemeinschaft and the second author by the Australian Research Council.

\section{REFERENCES}

[1] M. Ajtai, R. Kumar and D. Sivakumar, 'A sieve algorithm for the shortest lattice vector problem', Proc. 33rd ACM Symp. on Theory of Comput., ACM, 2001, 601-610. 9

[2] D. Boneh and R. Venkatesan, 'Hardness of computing the most significant bits of secret keys in Diffie-Hellman and related schemes', Advances in Cryptology - CRYPTO '96, Lect. Notes in Comp. Sci., Springer-Verlag, 1109 (1996), 129-142. 1, 2, 9, 10, 11, 12, 13

[3] D. Boneh and R. Venkatesan, 'Rounding in lattices and its cryptographic applications', Proc. 8th Annual ACM-SIAM Symp. on Discr. Algorithms, SIAM, 1997, 675-681. 1, 2, 9

[4] J. Bourgain, 'Estimates on polynomial exponential sums', Israel J. Math., 176 (2010), 221-240. 4

[5] Q. Cheng, S. Gao, J. M. Rojas and D. Wan, 'Counting roots for polynomials modulo prime powers', Proc. 13th Algorithmic Number Theory Symp., Open Book Ser., v.2, Math. Sci. Publ., Berkeley, CA, 2019, 191-205. 1

[6] H. Cheng and G. Labahn, 'Computing all factorizations in $\mathbb{Z}_{N}[x]$ ', Proc. 2001 ACM Intern. Symp. Symb. Algebraic Comp., ACM, New York, 2001, 64-71.

[7] T. Cochrane and Z. Zheng, 'Pure and mixed exponential sums, Acta Arith., 91 (1999), 249-278. 6

[8] J. H. Conway and N. J. A. Sloane, Sphere packings, lattices and groups, 3rd edition. Grundlehren der Mathematischen Wissenschaften, v.290, SpringerVerlag, New York, 1999. 9

[9] M. Drmota and R. Tichy, Sequences, discrepancies and applications, SpringerVerlag, Berlin, 1997. 6, 7

[10] A. Dwivedi, R. Mittal and N. Saxena, 'Counting basic-irreducible factors mod $p^{k}$ in deterministic poly-time and $p$-adic applications', Proc. 34th Comp. Compl. Conf., Leibniz Int. Proc. Inform., v.137, Schloss Dagstuhl. LeibnizZent. Inform., Wadern, 2019. Art. 15, 1-29. 1

[11] A. Dwivedi, R. Mittal and N. Saxena, 'Efficiently factoring polynomials modulo $p^{4}$, J. Symb. Comp., (to appear). 1

[12] O. Garcia-Morchon, R. Rietman, L. Tolhuizen and I. E. Shparlinski, 'Interpolation and approximation of polynomials in finite fields over a short interval from noisy values', Experimental Math., 23 (2014), 261-270. 2

[13] J. von zur Gathen and J. Gerhard, Modern computer algebra, Cambridge University Press, Cambridge, 2003. 1 
[14] J. von zur Gathen and S. Hartlieb, 'Factoring modular polynomials', J. Symb. Comp., 26 (1998), 583-606. 1

[15] J. von zur Gathen and I. E. Shparlinski, 'Polynomial Interpolation from multiples', Proc. 15th ACM-SIAM Symp. on Discr. Algorithms, SIAM, 2004, 209215. 14

[16] M. Grötschel, L. Lovász and A. Schrijver, Geometric algorithms and combinatorial optimization, Algorithms and Combinatorics: Study and Research Texts, v.2, Springer-Verlag, Berlin, 1993. 9

[17] P. M. Gruber and C. G. Lekkerkerker, 'Geometry of numbers', North-Holland Math. Library, v.37. North-Holland Publishing Co., Amsterdam, 1987. 9

[18] T. Hammonds, J. Johnson, A. Patini and R. M. Walker, 'Counting roots of polynomials over $\mathbb{Z} / p^{2} \mathbb{Z}$ ', Houston J. Math., 44 (2018), 1111-1119. 1

[19] R. Kannan, 'Algorithmic geometry of numbers', Annual Review of Comp. Sci., 2 (1987), 231-267. 9

[20] L. Kopp, N. Randall, J. M. Rojas, and Y. Zhu, 'Randomized polynomial-time root counting in prime power rings', Math. Comp., 89 (2020), 373-385. 1

[21] A. K. Lenstra, H. W. Lenstra and L. Lovász, 'Factoring polynomials with rational coefficients', Math. Ann., 261 (1982), 515-534. 9

[22] D. Micciancio and P. Voulgaris, 'A deterministic single exponential time algorithm for most lattice problems based on Voronoi cell computations', SIAM J. Comp., 42 (2013), 1364-1391. 9

[23] D. A. Mit'kin, 'Estimates and asymptotic formulas for rational exponential sums that are nearly complete', Math. USSR, 50 (1985), 513-532 (translated from Matem. Sbornik 122 (4), (1983), 527-545). 6

[24] P. Q. Nguyen, 'Public-key cryptanalysis', Recent Trends in Cryptography, Contemp. Math., v.477, Amer. Math. Soc., 2009, 67-119. 9

[25] P. Q. Nguyen and J. Stern, 'Lattice reduction in cryptology: An update', Proc. 13th Algorithmic Number Theory Symp., Lect. Notes in Comp. Sci., v.1838, Springer-Verlag, Berlin, 2000, 85-112. 9

[26] P. Q. Nguyen and J. Stern, 'The two faces of lattices in cryptology', Cryptography and Lattices, Lect. Notes in Comp. Sci., v.2146, Springer-Verlag, Berlin,2001, 146-180. 9

[27] O. Regev, 'On the complexity of lattice problems with polynomial approximation factors', The LLL Algorithm: Surveys and Applications, Springer-Verlag, 2010, 475-496. 9

[28] C. P. Schnorr, 'A hierarchy of polynomial time basis reduction algorithms', Theor. Comp. Sci., 53 (1987), 201-224. 9

[29] I. E. Shparlinski, 'On exponential sums with sparse polynomials and rational functions', J. Number Theory, 60 (1996), 233-244. 2, 4, 5, 6

[30] I. E. Shparlinski, 'Sparse polynomial approximation in finite fields', Proc. 33rd ACM Symp. on Theory of Comput., ACM, 2001, 209-215. 1, 2, 9, 10, 12

[31] I. E. Shparlinski, 'Playing "Hide-and-Seek" with numbers: The hidden number problem, lattices and exponential sums', Public-Key Cryptography, Proc. Symp. in Appl. Math., Amer. Math. Soc., Providence, RI, 62 (2005), 153-177. 2

[32] I. E. Shparlinski and A. Winterhof, 'Noisy interpolation of sparse polynomials in finite fields', Appl. Algebra in Engin., Commun. and Computing, 16 (2005), 307-317. 1, 2, 9, 10 
[33] C. Sircana, 'Factorization of polynomials over $\mathbb{Z} /\left(p^{n}\right)$ ', Proc. 2019 ACM Intern. Symp. Algebraic Comp., ACM, 2019, 405-412. 1

Department of Computer Science, Bonn University, 53113 Bonn, GERMANY

Email address: marek@cs.uni-bonn.de

Department of Pure Mathematics, University of New South Wales, Sydney, NSW 2052, Australia

Email address: igor.shparlinski@unsw.edu.au 